\title{
Computation of matrices and submodular functions values associated to finite topological spaces
}

\author{
Julian L. Cuevas Rozo ${ }^{1, *}$, Humberto Sarria Zapata ${ }^{1}$ \\ ${ }^{1}$ Dpto. de Matemáticas, Facultad de Ciencias, Universidad Nacional de Colombia, Bogotá, Colombia
}

\begin{abstract}
The submodular functions have shown their importance in the study and characterization of multiple properties of finite topological spaces, from numeric values provided by such functions (Sarria, Roa \& Varela, 2014). However, the calculation of these values has been performed manually or even using Hasse diagrams, which is not practical. In this article, we present some algorithms that let us calculate some kind of polymatroid functions, specifically $f_{\mathcal{U}}, f_{\mathcal{D}}, \bar{f}$ and $r_{A}$, which define a topology, by using topogenous matrices.
\end{abstract}

Key words: Finite topological spaces, submodular functions, topogenous matrix, Stong matrix.

Cálculo de matrices y funciones submodulares asociadas a espacios topológicos finitos

\section{Resumen}

Las funciones submodulares han mostrado su importancia en el estudio y la caracterización múltiples propiedades de los espacios topológicos finitos, a partir de valores numéricos proporcionados por dichas funciones (Sarria, Roa \& Varela, 2014). Sin embargo, el cálculo de éstos valores se ha realizado manualmente e incluso haciendo uso de diagramas de Hasse, lo que no es práctico. En este artículo, presentamos algunos algoritmos que nos permiten calcular cierta clase de funciones polimatroides, específicamente $f_{\mathcal{U}}, f_{\mathcal{D}}, \bar{f}$ y $r_{A}$, las cuales definen una topología, por medio del uso de matrices topogéneas.

Palabras clave: Espacios topológicos finitos, funciones submodulares, matriz topogénea, matriz de Stong.

\section{Introduction}

Alexandroff proved that finite topological spaces are in correspondence one-to-one with finite preorders (Alexandroff, 1937), showing that such spaces can be viewed from other mathematical structures. Likewise, Shiraki named as topogenous matrices the objects worked by Krishnamurthy, in an attempt to count the topologies that can be defined on a finite set (Krishnamurthy, 1966), a problem still unsolved. Moreover, such matrices provide all the information about the topology of a finite space (Shiraki, 1968), showing the relevance of these objects in the topological context.

Recently, connections between submodular functions and finite topological spaces have been developed (Abril, 2015), (Sarria et al., 2014), allowing to interpret many topological concepts through numeric values provided by such associated functions, which is really important if we want to mechanize the verification of topological properties on subsets of an arbitrary finite space.

In view of the above, we regard some matrices, as topogenous and Stong matrices defined in sections 2 and 3, which are useful, for example, in the study of lattice of all topologies on a fixed set, and we link such matrices with particular submodular functions $\left(f_{\mathcal{U}}, f_{\mathcal{D}}, \bar{f}\right.$ and $\left.r_{A}\right)$, important in the finite topological spaces context, by algorithms created to improve the computations shown in (Abril, 2015) and (Sarria et al., 2014).

\footnotetext{
*Corresponding autor:

Julian L. Cuevas Rozo, jlcuevasr@unal.edu.co

Received: September 30, 2016

Accepted: March 6, 2017
} 
From now on, we shall use exclusively the symbol $X$ to denote a set of $n$ elements $X=\left\{x_{1}, \ldots, x_{n}\right\}$, unless explicitly stated otherwise. We define $I_{n}=\{1,2, \ldots, n\}$ and for a permutation $\sigma$ of $I_{n}$, we use $P_{\sigma}$ to denote the matrix whose entries satisfy

$$
\left[P_{\sigma}\right]_{i j}=\delta(i, \sigma(j))
$$

where $\delta$ is the Kronecker delta.

\section{Topogenous matrix}

Given a finite topological space $(X, \mathscr{T})$, denote by $U_{k}$ the minimal open set containing $x_{k}$ :

$$
U_{k}=\bigcap_{x_{k} \in E \in \mathscr{T}} E
$$

and consider the colection $\mathcal{U}=\left\{U_{1}, \ldots, U_{n}\right\}$, which is the minimal basis for the space in the sense that $\mathcal{U}$ is contained in any other basis for the topology $\mathscr{T}$.

Definition 0.1. Let $(X, \mathscr{T})$ be a finite topological space and $\mathcal{U}=\left\{U_{1}, \ldots, U_{n}\right\}$ its minimal basis. The topogenous matrix $T_{X}=\left[t_{i j}\right]$ associated to $X$ is the square matrix of size $n \times n$ that satisfies:

$$
t_{i j}= \begin{cases}1 & , x_{i} \in U_{j} \\ 0 & , \text { in other case }\end{cases}
$$

Remark 0.2. (Shiraki, 1968) introduce the term topogenous matrix to denote the transpose matrix of that in above definition.

Example 0.3. In the next diagram, we represent the minimal basis for a topology on $X=\left\{x_{1}, x_{2}, x_{3}, x_{4}, x_{5}, x_{6}\right\}$. The minimal open sets are $U_{1}=U_{5}=\left\{x_{1}, x_{5}\right\}, U_{2}=$ $\left\{x_{2}, x_{4}\right\}, U_{3}=\left\{x_{3}\right\}, U_{4}=\left\{x_{4}\right\}, U_{6}=\left\{x_{4}, x_{6}\right\}$ and the associated topogenous matrix is as follows:

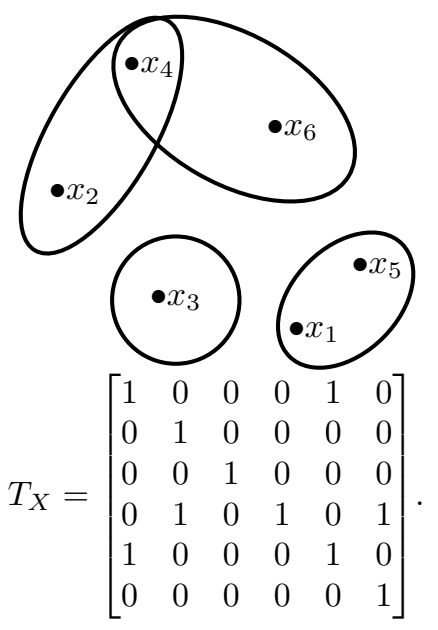

Example 0.4. Consider the topological space $(X, \mathscr{T})$ where $X=\{a, b, c, d, e\}$ and

$$
\begin{aligned}
\mathscr{T}=\{\emptyset,\{b\},\{d\},\{b, d\} & ,\{d, e\},\{b, d, e\},\{a, b, d\}, \\
& \{a, b, d, e\},\{a, b, c, d\}, X\}
\end{aligned}
$$

For the following orderings of the elements, we obtain the respective topogenous matrices as can be verified by calculating the minimal basis in each case:

$$
\begin{gathered}
\left(x_{1}, x_{2}, x_{3}, x_{4}, x_{5}\right)=(a, b, c, d, e) \\
T_{X_{1}}=\left[\begin{array}{lllll}
1 & 0 & 1 & 0 & 0 \\
1 & 1 & 1 & 0 & 0 \\
0 & 0 & 1 & 0 & 0 \\
1 & 0 & 1 & 1 & 1 \\
0 & 0 & 0 & 0 & 1
\end{array}\right] \\
\left(x_{1}, x_{2}, x_{3}, x_{4}, x_{5}\right)=(b, d, e, a, c) \\
T_{X_{2}}=\left[\begin{array}{lllll}
1 & 0 & 0 & 1 & 1 \\
0 & 1 & 1 & 1 & 1 \\
0 & 0 & 1 & 0 & 0 \\
0 & 0 & 0 & 1 & 1 \\
0 & 0 & 0 & 0 & 1
\end{array}\right]
\end{gathered}
$$

Topogenous matrices can be characterized by the following result.

Theorem 0.5. (Shiraki, 1968) Let $T=\left[t_{i j}\right]$ be the topogenous matrix associated to $(X, \mathscr{T})$. Then $T$ satisfies the following properties, for all $i, j, k \in I_{n}$ :

$$
\begin{aligned}
& \text { 1. } t_{i j} \in\{0,1\} . \\
& \text { 2. } t_{i i}=1 . \\
& \text { 3. } t_{i k}=t_{k j}=1 \Longrightarrow t_{i j}=1 \text {. }
\end{aligned}
$$

Conversely, if a square matrix $T=\left[t_{i j}\right]$ of size $n \times n$ satisfies the above properties, $T$ induces a topology on $X$.

Homeomorphism classes are also described by similarity via a permutation matrix between topogenous matrices.

Theorem 0.6. (Shiraki, 1968) Let $(X, \mathscr{T})$ and $(Y, \mathscr{H})$ be finite topological spaces with associated topogenous matrices $T_{X}$ and $T_{Y}$, respectively. Then $(X, \mathscr{T})$ and $(Y, \mathscr{H})$ are homeomorphic spaces if, and only if, $T_{X}$ and $T_{Y}$ are similar via a permutation matrix.

\section{Transiting $\operatorname{Top}(X)$}

Using topogenous matrices, we can find a way to transit through $\operatorname{Top}(X)$, the complete lattice of all topologies on a fixed set $X$. Given two topologies $\mathscr{T}_{1}$ and $\mathscr{T}_{2}$ in $\operatorname{Top}(X)$, the supremum of them, denoted by $\left\langle\mathscr{T}_{1} \cup \mathscr{T}_{2}\right\rangle$, is the topology whose open sets are unions of finite intersections of elements in the collection $\mathscr{T}_{1} \cup \mathscr{T}_{2}$. 
Proposition 0.7. (Cuevas, 2016) Let $\left(X, \mathscr{T}_{1}\right)$ and $\left(X, \mathscr{T}_{2}\right)$ be finite topological spaces with minimal basis $\mathcal{U}=\left\{U_{1}, \ldots, U_{n}\right\}$ and $\mathcal{V}=\left\{V_{1}, \ldots, V_{n}\right\}$, respectively. Then, the minimal basis $\mathcal{W}=\left\{W_{1}, \ldots, W_{n}\right\}$ for the space $\left(X, \mathscr{T}^{*}\right)$, where $\mathscr{T}^{*}=\left\langle\mathscr{T}_{1} \cup \mathscr{T}_{2}\right\rangle$, satisfies $W_{k}=U_{k} \cap V_{k}$ for all $k \in I_{n}$.

The next theorem shows a way to move ahead in $\operatorname{Top}(X)$, that is, it allows to find the supremum of two topologies. The symbol $\wedge$ is regarded in the following sense: if $E$ and $F$ are $n \times n$ matrices, $E \wedge F$ is the square matrix whose entries satisfy $[E \wedge F]_{i j}=\min \left\{[E]_{i j},[F]_{i j}\right\}$.

Theorem 0.8. Let $X_{1}=\left(X, \mathscr{T}_{1}\right), X_{2}=\left(X, \mathscr{T}_{2}\right)$ and $X^{*}=\left(X, \mathscr{T}^{*}\right)$ be finite topological spaces with topogenous matrices $T_{X_{1}}, T_{X_{2}}$ and $T_{X^{*}}$, respectively. If $\mathscr{T}^{*}=\left\langle\mathscr{T}_{1} \cup\right.$ $\left.\mathscr{T}_{2}\right\rangle$ then

$$
T_{X^{*}}=T_{X_{1}} \wedge T_{X_{2}}
$$

Conversely, if there exist finite spaces $X_{1}, X_{2}$ and $X^{*}$ which satisfy (1) then $\mathscr{T}^{*}=\left\langle\mathscr{T}_{1} \cup \mathscr{T}_{2}\right\rangle$.

Proof. Suppose that $\mathscr{T}^{*}=\left\langle\mathscr{T}_{1} \cup \mathscr{T}_{2}\right\rangle$ and fix an index $k \in I_{n}$. By proposition 0.7, we know that $W_{k}=U_{k} \cap V_{k}$, thus $x_{i} \in W_{k} \Longleftrightarrow x_{i} \in U_{k}$ and $x_{i} \in V_{k}$. Since the column $k$ of a topogenous matrix represents the minimal open set which contains $x_{k}$, then for each $i \in I_{n}$ it is satisfied that $\left[T_{X^{*}}\right]_{i k}=\min \left\{\left[T_{X_{1}}\right]_{i k},\left[T_{X_{2}}\right]_{i k}\right\}$.

The second part of the theorem holds by uniqueness of the minimal basis for a topology, since if (1) is satisfied, by the above argument we would have $T_{X^{*}}=T_{X_{1}} \wedge T_{X_{2}}=$ $T_{\left(X,\left\langle\mathscr{T}_{1} \cup \mathscr{T}_{2}\right\rangle\right)}$ and thus $\mathscr{T}^{*}=\left\langle\mathscr{T}_{1} \cup \mathscr{T}_{2}\right\rangle$.

A way to go back in $\operatorname{Top}(X)$ is finding subtopologies, which is possible using topogenous matrices as is shown in the next result.

Corollary 0.9. Let $X_{1}=\left(X, \mathscr{T}_{1}\right)$ and $X_{2}=\left(X, \mathscr{T}_{2}\right)$ be finite topological spaces. Then $\mathscr{T}_{1} \subseteq \mathscr{T}_{2}$ if, and only if, $\left[T_{X_{2}}\right]_{i j} \leqslant\left[T_{X_{1}}\right]_{i j}$ for all $i, j \in I_{n}$.

Proof. The result follows from the next chain of equivalences:

$$
\begin{aligned}
\mathscr{T}_{1} \subseteq \mathscr{T}_{2} & \Longleftrightarrow \mathscr{T}_{2}=\left\langle\mathscr{T}_{1} \cup \mathscr{T}_{2}\right\rangle \\
& \Longleftrightarrow T_{X_{2}}=T_{X_{1}} \wedge T_{X_{2}} \Longleftrightarrow\left[T_{X_{2}}\right]_{i j} \leqslant\left[T_{X_{1}}\right]_{i j}
\end{aligned}
$$

\section{Triangularization of topogenous matrices}

Let $(X, \mathscr{T})$ be a finite topological space with minimal basis $\mathcal{U}=\left\{U_{1}, \ldots, U_{n}\right\}$ and associated topogenous matrix $T_{X}=$ $\left[t_{i j}\right]$. Define the binary relation $\leqslant$ on $X$ as follows:

$$
x_{i} \leqslant x_{j} \Longleftrightarrow x_{i} \in U_{j} \Longleftrightarrow U_{i} \subseteq U_{j} \Longleftrightarrow t_{i j}=1
$$

This relation is a preorder on the space, that is, it is reflexive and transitive. The next theorem shows under which condition such a relation is a partial order on $X$.

Theorem 0.10. (Alexandroff, 1937) Topologies on a finite set $X$ are in one-to-one correspondence with preorders on $X$. Moreover, a finite topological space $(X, \mathscr{T})$ is $T_{0}$ if, and only if, $(X, \leqslant)$ is a poset.

Example 0.11. Consider the space $(X, \mathscr{T})$ given in the example 0.4 with the ordering

$$
\left(x_{1}, x_{2}, x_{3}, x_{4}, x_{5}\right)=(a, b, c, d, e) \text {. }
$$

Such space satisfies the $T_{0}$ axiom. Hasse diagram of the poset $(X, \leqslant)$ is the next:

$$
\begin{aligned}
& U_{1}=\left\{x_{1}, x_{2}, x_{4}\right\} \\
& U_{2}=\left\{x_{2}\right\} \\
& U_{3}=\left\{x_{1}, x_{2}, x_{3}, x_{4}\right\} \\
& U_{4}=\left\{x_{4}\right\} \\
& U_{5}=\left\{x_{4}, x_{5}\right\}
\end{aligned}
$$

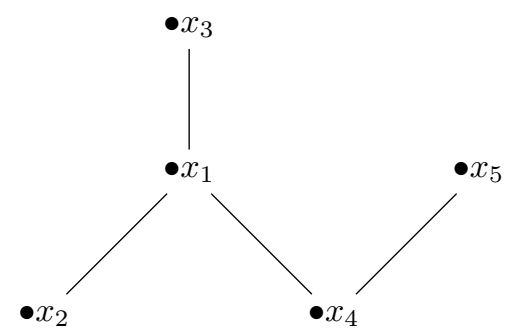

In the example 0.4 , it was possible to associate an upper triangular topogenous matrix to the considered space since such topological space is $T_{0}$, as shows the next Shiraki's theorem.

Theorem 0.12. (Shiraki, 1968) A finite topological space $(X, \mathscr{T})$ is $T_{0}$ if, and only if, its associated topogenous matrix $T_{X}$ is similar via a permutation matrix to an upper triangular topogenous matrix.

A procedure to triangularize a topogenous matrix of a $T_{0}$ space $X$ is described below. Given a topogenous matrix $T_{X}=\left[t_{i j}\right]$ define $M_{k}=\sum_{i=1}^{n} t_{i k}=\left|U_{k}\right|$, for each $k \in I_{n}$; if we organize them in ascending order

$$
M_{k_{1}} \leqslant M_{k_{2}} \leqslant \cdots \leqslant M_{k_{n}}
$$

and consider the permutation $\sigma=\left(\begin{array}{cccc}1 & 2 & \cdots & n \\ k_{1} & k_{2} & \cdots & k_{n}\end{array}\right)$, the topogenous matrix $P_{\sigma}^{\mathrm{T}} T_{X} P_{\sigma}$ is upper triangular: if $i>j$ and $t_{\sigma(i) \sigma(j)}=1$, we would have $x_{\sigma(i)}<x_{\sigma(j)}$ and therefore $M_{k_{i}}<M_{k_{j}}$ which contradicts the ordering of $M_{k}$, hence $t_{\sigma(i) \sigma(j)}=0$. 
Example 0.13. Consider the topological space $X$, represented by the next Hasse diagram and topogenous matrix:

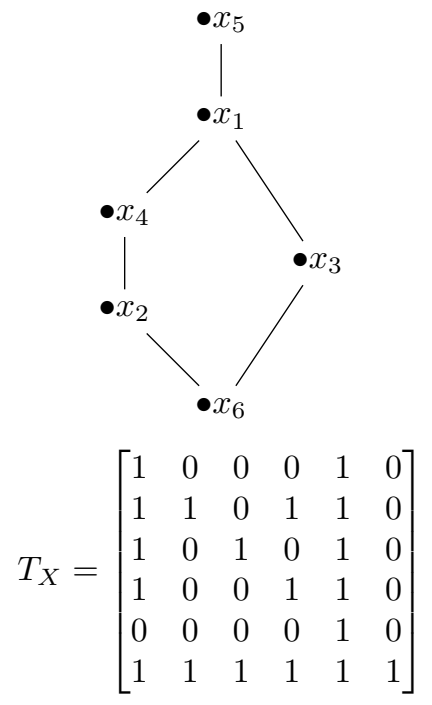

First, we find that: $M_{1}=5, M_{2}=M_{3}=2, M_{4}=3, M_{5}=$ 6 and $M_{6}=1$. Therefore we obtain the permutation

$$
\sigma=\left(\begin{array}{llllll}
1 & 2 & 3 & 4 & 5 & 6 \\
6 & 2 & 3 & 4 & 1 & 5
\end{array}\right)=(165)
$$

whose associated matrix is given by

$$
P_{\sigma}=\left[\begin{array}{llllll}
0 & 0 & 0 & 0 & 1 & 0 \\
0 & 1 & 0 & 0 & 0 & 0 \\
0 & 0 & 1 & 0 & 0 & 0 \\
0 & 0 & 0 & 1 & 0 & 0 \\
0 & 0 & 0 & 0 & 0 & 1 \\
1 & 0 & 0 & 0 & 0 & 0
\end{array}\right] .
$$

If we denote by $\widehat{x}_{k}=x_{\sigma(k)}$, the new ordering of the elements is represented in the topogenous matrix as follows:

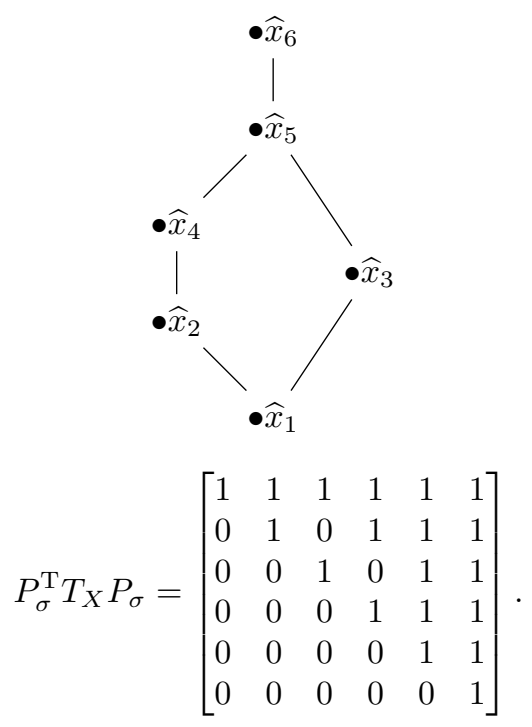

Remark 0.14. Observe that, in general, the permutation $\sigma$ constructed using the relations in (3) is not unique. In example 0.13 , we could have used $\sigma^{\prime}=(165)(23)$ (and, in this case, no other!) to triangularize $T_{X}$ obtaining the upper triangular matrix:

$$
P_{\sigma^{\prime}}^{\mathrm{T}} T_{X} P_{\sigma^{\prime}}=\left[\begin{array}{llllll}
1 & 1 & 1 & 1 & 1 & 1 \\
0 & 1 & 0 & 0 & 1 & 1 \\
0 & 0 & 1 & 1 & 1 & 1 \\
0 & 0 & 0 & 1 & 1 & 1 \\
0 & 0 & 0 & 0 & 1 & 1 \\
0 & 0 & 0 & 0 & 0 & 1
\end{array}\right] .
$$

Despite of this lack of uniqueness to choose such permutation $\sigma$, the resulting $T_{0}$ spaces are always homeomorphic (Theorem 0.6), so the topological properties are the same.

Remark 0.15. From now on, when $(X, \mathscr{T})$ is a $T_{0}$ space, we assume a fixed ordering in the elements of $X$ such that its topogenous matrix $T_{X}$ is upper triangular.

\section{Stong matrix}

Definition 0.16. Given $X$ a $T_{0}$ space, we define the Stong matrix $S_{X}=\left[s_{i j}\right]$ as the square matrix of size $n \times n$ that satisfies

$$
s_{i j}= \begin{cases}1 & , x_{i} \leqslant x_{j} \text { and there is no } k \text { with } x_{i}<x_{k}<x_{j} \\ 0 & , \text { in other case. }\end{cases}
$$

A simple method to calculate the topogenous matrix $T_{X}$ and the Stong matrix $S_{X}$ of the space $X$, from the associated Hasse diagram, is described below. Number the vertices so that $x_{i}<x_{j} \Longrightarrow i<j$, that is, number them from bottom to top ensuring that the topogenous matrix is upper triangular. For each $i \neq j$ :

- $t_{i j}=1$ if, and only if, there exists a chain whose minimum is $x_{i}$ and maximum is $x_{j}$.

- $s_{i j}=1$ if, and only if, $\left(x_{i}, x_{j}\right)$ is an edge of the diagram.

Remark 0.17. $t_{i j}=0 \Rightarrow s_{i j}=0$ and $s_{i j}=1 \Rightarrow t_{i j}=1$.

Example 0.18. Consider the Hasse diagram (left) numbering its vertices as described before (right):

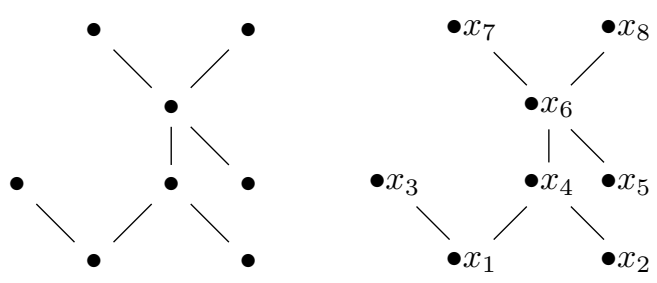


For example, for $x_{5}$ we have $x_{5} \leqslant x_{5}, x_{5} \leqslant x_{6}, x_{5} \leqslant x_{7}$ and $x_{5} \leqslant x_{8}$ then the fifth row of $T_{X}$ is $\left[\begin{array}{lllllll}0 & 0 & 0 & 0 & 1 & 1 & 1\end{array}\right]$. Also, the edges with initial point $x_{1}$ are $\left(x_{1}, x_{3}\right)$ and $\left(x_{1}, x_{4}\right)$, then the first row of $S_{X}$ is $\left[\begin{array}{lllllll}1 & 0 & 1 & 1 & 0 & 0 & 0\end{array}\right]$. The associated matrices are the following:

$$
\begin{aligned}
T_{X} & =\left[\begin{array}{llllllll}
1 & 0 & 1 & 1 & 0 & 1 & 1 & 1 \\
0 & 1 & 0 & 1 & 0 & 1 & 1 & 1 \\
0 & 0 & 1 & 0 & 0 & 0 & 0 & 0 \\
0 & 0 & 0 & 1 & 0 & 1 & 1 & 1 \\
0 & 0 & 0 & 0 & 1 & 1 & 1 & 1 \\
0 & 0 & 0 & 0 & 0 & 1 & 1 & 1 \\
0 & 0 & 0 & 0 & 0 & 0 & 1 & 0 \\
0 & 0 & 0 & 0 & 0 & 0 & 0 & 1
\end{array}\right] \\
S_{X} & =\left[\begin{array}{llllllll}
1 & 0 & 1 & 1 & 0 & 0 & 0 & 0 \\
0 & 1 & 0 & 1 & 0 & 0 & 0 & 0 \\
0 & 0 & 1 & 0 & 0 & 0 & 0 & 0 \\
0 & 0 & 0 & 1 & 0 & 1 & 0 & 0 \\
0 & 0 & 0 & 0 & 1 & 1 & 0 & 0 \\
0 & 0 & 0 & 0 & 0 & 1 & 1 & 1 \\
0 & 0 & 0 & 0 & 0 & 0 & 1 & 0 \\
0 & 0 & 0 & 0 & 0 & 0 & 0 & 1
\end{array}\right]
\end{aligned}
$$

\section{Relation between topogenous and Stong matrices}

We can reconstruct the topogenous matrix from the Stong matrix and vice versa. In the first case, using theorem 0.5 , we see that the topogenous matrix is the incidence matrix of the transitive closure of the binary relation represented by the Stong matrix. For the matrix given in example 0.18

$$
S_{X}=\left[s_{i j}\right]=\left[\begin{array}{cccccccc}
1 & 0 & 1 & 1 & 0 & 0 & 0 & 0 \\
0 & 1 & 0 & 1 & 0 & 0 & 0 & 0 \\
0 & 0 & 1 & 0 & 0 & 0 & 0 & 0 \\
0 & 0 & 0 & 1 & 0 & 1 & 0 & 0 \\
0 & 0 & 0 & 0 & 1 & 1 & 0 & 0 \\
0 & 0 & 0 & 0 & 0 & 1 & 1 & 1 \\
0 & 0 & 0 & 0 & 0 & 0 & 1 & 0 \\
0 & 0 & 0 & 0 & 0 & 0 & 0 & 1
\end{array}\right]
$$

we add ones in each upper subdiagonal. The first subdiagonal, consisting of the elements $s_{k, k+1}, 1 \leqslant k \leqslant 7$, is not modified because if there is a $k$ such that $s_{k, k+1}=0$ and $t_{k, k+1}=1$, there would exist $x_{j}$ with $x_{k}<x_{j}<x_{k+1}$, which is not possible since the ordering we have chosen in remark 0.15 would imply $k<j<k+1$, a contradiction. For the second subdiagonal, whose elements are $s_{k, k+2}$, $1 \leqslant k \leqslant 6$, there are zeros in the entries $s_{35}$ and $s_{57}$. Since $s_{56}=s_{67}=1$ we have $t_{56}=t_{67}=1 \Longrightarrow t_{57}=1$ (theorem $0.5)$ and thus we add a one in the entry $(5,7)$. In the case $s_{35}=0$ there is no modification, because $s_{34}=s_{45}=0$ :

$$
S_{X}^{(2)}=\left[s_{i j}^{(2)}\right]=\left[\begin{array}{cccccccc}
1 & 0 & 1 & 1 & 0 & 0 & 0 & 0 \\
0 & 1 & 0 & 1 & 0 & 0 & 0 & 0 \\
0 & 0 & 1 & 0 & 0 & 0 & 0 & 0 \\
0 & 0 & 0 & 1 & 0 & 1 & 0 & 0 \\
0 & 0 & 0 & 0 & 1 & 1 & 1 & 0 \\
0 & 0 & 0 & 0 & 0 & 1 & 1 & 1 \\
0 & 0 & 0 & 0 & 0 & 0 & 1 & 0 \\
0 & 0 & 0 & 0 & 0 & 0 & 0 & 1
\end{array}\right]
$$

For the third subdiagonal, with elements $s_{k, k+3}, 1 \leqslant k \leqslant$ 5 , we have zeros in the entries $s_{25}^{(2)}, s_{36}^{(2)}, s_{47}^{(2)}$ and $s_{58}^{(2)}$. For example, since $s_{46}^{(2)}=s_{67}^{(2)}=1$ then $t_{46}=t_{67}=1 \Longrightarrow t_{47}=$ 1 , and hence we add a one in the entry $(4,7)$. Similarly for the entry $(5,8)$ since $s_{56}^{(2)}=s_{68}^{(2)}=1$. Entry $(2,5)$ is not changed because there exists no $k$ such that $s_{2 k}^{(2)}=$ $s_{k 5}^{(2)}=1$, and for the same reason we do not modify the entry $(3,6)$ :

$$
S_{X}^{(3)}=\left[s_{i j}^{(3)}\right]=\left[\begin{array}{llllllll}
1 & 0 & 1 & 1 & 0 & 0 & 0 & 0 \\
0 & 1 & 0 & 1 & 0 & 0 & 0 & 0 \\
0 & 0 & 1 & 0 & 0 & 0 & 0 & 0 \\
0 & 0 & 0 & 1 & 0 & 1 & \mathbf{1} & 0 \\
0 & 0 & 0 & 0 & 1 & 1 & 1 & \mathbf{1} \\
0 & 0 & 0 & 0 & 0 & 1 & 1 & 1 \\
0 & 0 & 0 & 0 & 0 & 0 & 1 & 0 \\
0 & 0 & 0 & 0 & 0 & 0 & 0 & 1
\end{array}\right]
$$

Proceeding similarly for the following subdiagonals, we obtain the matrices $S_{X}^{(4)}, S_{X}^{(5)}, S_{X}^{(6)}$ and $S_{X}^{(7)}$ :

$$
\begin{aligned}
S_{X}^{(4)} & =\left[\begin{array}{llllllll}
1 & 0 & 1 & 1 & 0 & 0 & 0 & 0 \\
0 & 1 & 0 & 1 & 0 & \mathbf{1} & 0 & 0 \\
0 & 0 & 1 & 0 & 0 & 0 & 0 & 0 \\
0 & 0 & 0 & 1 & 0 & 1 & 1 & \mathbf{1} \\
0 & 0 & 0 & 0 & 1 & 1 & 1 & 1 \\
0 & 0 & 0 & 0 & 0 & 1 & 1 & 1 \\
0 & 0 & 0 & 0 & 0 & 0 & 1 & 0 \\
0 & 0 & 0 & 0 & 0 & 0 & 0 & 1
\end{array}\right] \\
S_{X}^{(5)} & =\left[\begin{array}{llllllll}
1 & 0 & 1 & 1 & 0 & \mathbf{1} & 0 & 0 \\
0 & 1 & 0 & 1 & 0 & 1 & \mathbf{1} & 0 \\
0 & 0 & 1 & 0 & 0 & 0 & 0 & 0 \\
0 & 0 & 0 & 1 & 0 & 1 & 1 & 1 \\
0 & 0 & 0 & 0 & 1 & 1 & 1 & 1 \\
0 & 0 & 0 & 0 & 0 & 1 & 1 & 1 \\
0 & 0 & 0 & 0 & 0 & 0 & 1 & 0 \\
0 & 0 & 0 & 0 & 0 & 0 & 0 & 1
\end{array}\right] \\
S_{X}^{(6)} & =\left[\begin{array}{llllllll}
1 & 0 & 1 & 1 & 0 & 1 & \mathbf{1} & 0 \\
0 & 1 & 0 & 1 & 0 & 1 & 1 & \mathbf{1} \\
0 & 0 & 1 & 0 & 0 & 0 & 0 & 0 \\
0 & 0 & 0 & 1 & 0 & 1 & 1 & 1 \\
0 & 0 & 0 & 0 & 1 & 1 & 1 & 1 \\
0 & 0 & 0 & 0 & 0 & 1 & 1 & 1 \\
0 & 0 & 0 & 0 & 0 & 0 & 1 & 0 \\
0 & 0 & 0 & 0 & 0 & 0 & 0 & 1
\end{array}\right]
\end{aligned}
$$




$$
S_{X}^{(7)}=\left[\begin{array}{llllllll}
1 & 0 & 1 & 1 & 0 & 1 & 1 & \mathbf{1} \\
0 & 1 & 0 & 1 & 0 & 1 & 1 & 1 \\
0 & 0 & 1 & 0 & 0 & 0 & 0 & 0 \\
0 & 0 & 0 & 1 & 0 & 1 & 1 & 1 \\
0 & 0 & 0 & 0 & 1 & 1 & 1 & 1 \\
0 & 0 & 0 & 0 & 0 & 1 & 1 & 1 \\
0 & 0 & 0 & 0 & 0 & 0 & 1 & 0 \\
0 & 0 & 0 & 0 & 0 & 0 & 0 & 1
\end{array}\right]
$$

Since we reached the last upper subdiagonal, we obtain the topogenous matrix $T_{X}=S_{X}^{(7)}$, which coincides with the matrix of example 0.18 .

The above procedure can be applied to any finite $T_{0}$ space $X$ by using the following algorithm.

Algorithm: Topogenous matrix from Stong matrix

Input: Stong matrix $S_{X}=\left[s_{i j}\right]_{n \times n}$ associated to the $T_{0}$ space $X$.

Output: Topogenous matrix $T_{X}=\left[t_{i j}\right]_{n \times n}$ associated to the $T_{0}$ space $X$.

1. if $n=1,2$ do $T=S$, else

2. if $j \leqslant i+1$ or $s_{i j}=1$ do $t_{i j}=s_{i j}$, else

3. for $j=3 \cdots n$

4. for each zero element $s_{i, i+j-1}$ in the $j$-th upper subdiagonal

5. $\quad$ if there exists $r$ such that $s_{i r}=1=$ $s_{r, i+j-1}$ do $t_{i, i+j-1}=1$

else $t_{i, i+j-1}=0$.

6. end for

7. end for

Reversing the above process, we obtain an algorithm to find the Stong matrix knowing the topogenous matrix of the space.

Algorithm: Stong matrix from topogenous matrix

Input: Topogenous matrix $T_{X}=\left[t_{i j}\right]_{n \times n}$ associated to the $T_{0}$ space $X$.

Output: Stong matrix $S_{X}=\left[s_{i j}\right]_{n \times n}$ associated to the $T_{0}$ space $X$.

1. for $i=1 \cdots n$
2. for $j=1 \cdots n$

3. if $j \leqslant i+1$ or $t_{i j}=0$ do $s_{i j}=t_{i j}$, else

4. if there exists $k$, between $i+1$ and $j-1$, such that $t_{i k}=1=t_{k j}$

$$
\text { do } s_{i j}=0 \text {, else } s_{i j}=1
$$

\section{5. end for}

6. end for

\section{Calculating submodular functions values}

\section{The entropy function and $\bar{f}$}

The entropy function $r_{A}$ defined on an arbitrary FDrelation is studied in (Sarria et al., 2014), in an attempt to apply the information theory in different structures. For each $\mathcal{N}$ FD-relation, there exists an special set $A$ such that $r_{A} \in \lambda(\mathcal{N})$, which in our particular case of topological FD-relations, allows to find a polymatroid that characterizes the topological properties of the space, as is shown in (Sarria et al., 2014). Here $\lambda(\mathcal{N})$ is the set of submodular and non-decreasing functions such that $\mathcal{N}_{f}=\mathcal{N}$. We intend in this subsection to propose an algorithm to find the values of such entropy function in any subset.

We know that $E=\left\{x_{i_{1}}, \ldots, x_{i_{r}}\right\} \subseteq X$ is a closed set if, and only if, it coincides with its closure which means that every adherent point of $E$ is in $E$. Using the minimal basis $\mathcal{U}=\left\{U_{x}\right\}_{x \in X}$ of the space $X$, we have the following characterization:

$E$ is a closed set of $X \Longleftrightarrow U_{x} \cap E=\emptyset$ for all $x \notin E$

Bearing in mind that each column $k$ of the topogenous matrix represents the minimal open set $U_{k}$, we shall find one by one the adherent points of $E$, not in $E$, finding the smallest closed set $C$ which contains it, using the characterization given in (4), by the following procedure: we take $C^{(1)}:=E=\left\{x_{i_{1}}, \ldots, x_{i_{r}}\right\}$; for each $x_{k}$ such that $k \notin\left\{i_{1}, \ldots, i_{r}\right\}$, we consider the $k$-th column of the topogenous matrix and check the elements in rows $i_{1}, \ldots, i_{r}$ in such column; if they are all zero, we take $C=C^{(1)}$, otherwise, there would exist $j_{1}$ such that $x_{j_{1}}$ is an adherent point of $E$ hence we take $C^{(2)}=E \cup\left\{x_{j_{1}}\right\}$. Now, for each $x_{k}$ such that $k \notin\left\{i_{1}, \ldots, i_{r}, j_{1}\right\}$, we consider the $k$-th column of the topogenous matrix and check the elements in rows $i_{1}, \ldots, i_{r}, j_{1}$ in such column; if they are all zero, we take $C=C^{(2)}$, otherwise, there would exist $j_{2}$ such that $x_{j_{2}}$ is an adherent point of $E$, hence we take $C^{(3)}=E \cup\left\{x_{j_{1}}, x_{j_{2}}\right\}$. 
Proceeding recursively, we continue adding points to obtain a set $C^{(m)}=E \cup\left\{x_{j_{1}}, x_{j_{2}}, \ldots, x_{j_{m-1}}\right\}$ such that $C^{(m)}=X$, in which case the set $E$ is dense in $X$, or $C^{(m)}$ is such that for all $k \notin\left\{i_{1}, \ldots, i_{r}, j_{1}, \ldots, j_{m-1}\right\}$, the $k$-th column has zeros in entries $i_{1}, \ldots, i_{r}, j_{1}, \ldots, j_{m-1}$, in which case $C=C^{(m)}$.

Example 0.19. We shall find the closure of $\left\{x_{2}, x_{4}\right\}$ in the topological space $X$ whose topogenous matrix is the following:

$$
T_{X}=\left[\begin{array}{lllll}
1 & 0 & 1 & 0 & 0 \\
0 & 1 & 1 & 1 & 0 \\
0 & 0 & 1 & 0 & 0 \\
0 & 0 & 0 & 1 & 0 \\
0 & 0 & 0 & 0 & 1
\end{array}\right] .
$$

Initially, we take $C^{(1)}=\left\{x_{2}, x_{4}\right\}$. Since the first column has zeros in rows 2 and 4 , the element $x_{1}$ is not added to $C^{(1)}$; the third column has a one in the second row and so we make $C^{(2)}=\left\{x_{2}, x_{3}, x_{4}\right\}$. Every time we add a point, we must look again at those elements that we had discarded in the previous step, in this case $x_{1}$. The first column has zeros in rows 2,3 and 4 , then $x_{1}$ is not added to $C^{(2)}$; finally the fifth column has zeros in 2,3 and 4 , so $x_{5}$ is not added to $C^{(2)}$, and as we have exhausted all points of space, we conclude that the closure of $\left\{x_{2}, x_{4}\right\}$ is $C=C^{(2)}=\left\{x_{2}, x_{3}, x_{4}\right\}$.

The above comments help find the closure of an arbitrary subset using the following algorithm.

Algorithm: Closure of A SUBSET $I \subseteq X$

Input: Topogenous matrix $T_{X}=\left[t_{i j}\right]_{n \times n}$ associated to the space $X$, a subset $I \subseteq X$.

Output: Closure of $I: c(I)=C$.

1. Define $C=I$ and $r=1$.

2. while $r=1$

3. for $x_{i} \notin C$

4. if $\sum_{x_{k} \in C} t_{i k} \neq 0$ do $C \leftarrow C \cup\left\{x_{i}\right\}$ and go to step 3.

5. if $i=\max \left\{j: x_{j} \notin C\right\}$ do $r=0$.

6. end for

7. end while
Remark 0.20. In (Sarria et al., 2014) is proved that the map

$$
\begin{aligned}
\bar{f}: 2^{X} & \longrightarrow \mathbb{Z} \\
I & \longrightarrow \bar{f}(I)=|c(I)|
\end{aligned}
$$

where $c$ is the closure operator associated to the finite topological space $(X, \mathscr{T})$, is a polymatroid which satisfies $\bar{f} \in \lambda\left(\mathcal{N}_{\mathscr{T}}\right)$ hence, in particular, the above algorithm allows to determine the function values of $\bar{f}$ completely.

Now, let $n_{K}=|c(K)|$ be the cardinal number of the closure of $K$. In (Sarria et al., 2014) it is shown that if $F_{K}=2^{n}-2^{n_{K}}$, the entropy function $r_{A}: 2^{X} \longrightarrow \mathbb{R}$ satisfies

$$
r_{A}(I)=\ln |A|-\frac{2 S_{I}}{|A|} \ln 2
$$

where

$$
\begin{aligned}
S_{I} & =\sum_{I \subseteq c(K)} F_{K} \\
M_{I} & =\sum_{I \nsubseteq c(K)} F_{K} \\
|A| & =2\left(M_{I}+S_{I}\right)
\end{aligned}
$$

We can rewrite $S_{I}$ and $M_{I}$ as follows:

$$
\begin{aligned}
S_{I}=\sum_{I \subseteq c(K)} F_{K} & =\sum_{I \subseteq c(K)}\left(2^{n}-2^{n_{K}}\right) \\
& =2^{n} \sum_{I \subseteq c(K)}\left[1-2^{n_{K}-n}\right]=: 2^{n} S_{I}^{*} \\
M_{I}=\sum_{I \nsubseteq c(K)} F_{K} & =\sum_{I \nsubseteq c(K)}\left(2^{n}-2^{n_{K}}\right) \\
& =2^{n} \sum_{I \nsubseteq c(K)}\left[1-2^{n_{K}-n}\right]=: 2^{n} M_{I}^{*}
\end{aligned}
$$

so that $|A|=2^{n+1}\left(M_{I}^{*}+S_{I}^{*}\right)$ and hence

$$
\begin{aligned}
r_{A}(I) & =\ln |A|-\frac{2 S_{I}}{|A|} \ln 2 \\
& =\ln \left(2^{n+1}\left(M_{I}^{*}+S_{I}^{*}\right)\right)-\frac{2^{n+1} S_{I}^{*}}{2^{n+1}\left(M_{I}^{*}+S_{I}^{*}\right)} \ln 2 \\
& =(n+1) \ln 2+\ln \left(M_{I}^{*}+S_{I}^{*}\right)-\frac{S_{I}^{*}}{\left(M_{I}^{*}+S_{I}^{*}\right)} \ln 2 \\
& =\ln \left(M_{I}^{*}+S_{I}^{*}\right)+\left[n+\frac{M_{I}^{*}}{M_{I}^{*}+S_{I}^{*}}\right] \ln 2
\end{aligned}
$$


Having the algorithm to find the closure of a subset $K$, and therefore to calculate the values $n_{K}, S_{I}^{*}$ and $M_{I}^{*}$, we can evaluate the function $r_{A}$ by the following algorithm.

Algorithm:Entropy fUnCtion $r_{A}$ VALUE in a SUBSET $I \subseteq$ $X$

Input: Topogenous matrix $T_{X}=\left[t_{i j}\right]_{n \times n}$ associated to the space $X$, a subset $I \subseteq X$.

Output: Value $r_{A}(I)$.

1. Define $M_{I}^{*}=S_{I}^{*}=0$.

2. for $K \subseteq X$

3. Calculate $n_{K}=|c(K)|$.

4. $\quad$ if $I \subseteq c(K)$ do $S_{I}^{*} \leftarrow S_{I}^{*}+1-2^{n_{K}-n}$ else $M_{I}^{*} \leftarrow M_{I}^{*}+1-2^{n_{K}-n}$

5. end for

6. Calculate $r_{A}(I)=\ln \left(M_{I}^{*}+S_{I}^{*}\right)+\left[n+\frac{M_{I}^{*}}{M_{I}^{*}+S_{I}^{*}}\right] \ln 2$

\section{Functions $f_{\mathcal{U}}$ and $f_{\mathcal{D}}$ : matrices $U_{X}$ and $D_{X}$}

Let $(X, \mathscr{T})$ be a finite topological space, $\mathcal{U}$ its minimal basis and $\mathcal{D}$ the minimal basis for $X^{o p}$, the opposite space of $X$, and consider the non-decreasing submodular function $f_{\Delta}: 2^{X} \longrightarrow \mathbb{Z}$ defined by

$$
f_{\Delta}(I):=\sum_{J \in \Delta} q_{J}(I)
$$

where $\Delta \subseteq 2^{X}$ and $q_{J}: 2^{X} \rightarrow\{0,1\}$ is the map which satisfies

$$
q_{J}(I):= \begin{cases}1, & I \nsubseteq J \\ 0, & I \subseteq J\end{cases}
$$

for each $I, J \subseteq X$. In (Abril, 2015) is proved that if $B$ is a subset of $X$ then

$$
\begin{aligned}
& f_{\mathcal{U}}(B)=|X|-\left|B^{\nabla}\right| \\
& f_{\mathcal{D}}(B)=|X|-\left|B_{\triangle}\right|
\end{aligned}
$$

where $B^{\nabla}$ and $B_{\triangle}$ denote the sets of upper and lower bounds of $B$ in $(X, \leqslant)$, respectively.

These functions $f_{\mathcal{U}}$ and $f_{\mathcal{D}}$ are important to describe the topology of the space, since they characterize the order relation $\leqslant$ given in $(2)$ :

$$
x_{i} \leqslant x_{j} \Longleftrightarrow f_{\mathcal{D}}\left(x_{i}\right)=f_{\mathcal{D}}\left(x_{i}, x_{j}\right)
$$

$$
x_{i} \leqslant x_{j} \Longleftrightarrow f_{\mathcal{U}}\left(x_{j}\right)=f_{\mathcal{U}}\left(x_{i}, x_{j}\right)
$$

Definition 0.21. Given a finite topological space $X$, we define the matrix $U_{X}=\left[u_{i j}\right]$ associated to the function $f_{\mathcal{U}}$, as the matrix which satisfies $u_{i j}=f_{\mathcal{U}}\left(x_{i}, x_{j}\right)$. Analogously, we define the matrix $D_{X}=\left[d_{i j}\right]$ associated to the function $f_{\mathcal{D}}$ as the matrix satisfying $d_{i j}=f_{\mathcal{D}}\left(x_{i}, x_{j}\right)$.

Proposition 0.22. If $T_{X}$ is the topogenous matrix associated to the finite topological space $X$, then

$$
\begin{aligned}
& U_{X}=n \mathbf{1}-T_{X} T_{X}^{\mathrm{T}} \\
& D_{X}=n \mathbf{1}-T_{X}^{\mathrm{T}} T_{X}
\end{aligned}
$$

where $\mathbf{1}=\left[a_{i j}\right]$ is the square matrix of size $n \times n$ such that $a_{i j}=1$ for all $i, j \in I_{n}$.

Proof. If $E=\left\{x_{i}, x_{j}\right\}^{\nabla}$, we show that $|E|=\sum_{k=1}^{n} t_{i k} t_{j k}$ : in fact, we have the equivalences:

$$
\begin{aligned}
x_{k} \in E & \Longleftrightarrow x_{i} \leqslant x_{k} y x_{j} \leqslant x_{k} \\
& \Longleftrightarrow t_{i k}=1 y t_{j k}=1 \\
& \Longleftrightarrow t_{i k} t_{j k}=1
\end{aligned}
$$

then each $x_{k} \in E$ provides a one in the sum $\sum_{k=1}^{n} t_{i k} t_{j k}$, thus having the required equality. Now, if $T_{X} T_{X}^{\overline{\mathrm{T}}}=\left[c_{i j}\right]$ then $c_{i j}=\sum_{k=1}^{n} t_{i k} t_{j k}=|E|$ and hence

$$
u_{i j}=f_{\mathcal{U}}\left(x_{i}, x_{j}\right)=|X|-|E|=n-c_{i j}=\left[n \mathbf{1}-T_{X} T_{X}^{\mathrm{T}}\right]_{i j} .
$$

Let $A=\left\{x_{i}, x_{j}\right\}_{\triangle}$. By a similar argument above, we have $|A|=\sum_{k=1}^{n} t_{k i} t_{k j}$ from which we get

$$
d_{i j}=f_{\mathcal{D}}\left(x_{i}, x_{j}\right)=|X|-|A|=\left[n \mathbf{1}-T_{X}^{\mathrm{T}} T_{X}\right]_{i j} .
$$

Example 0.23. For the next $T_{0}$ space

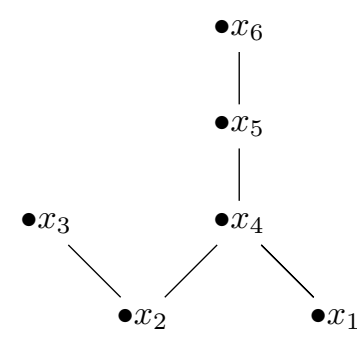

$$
T_{X}=\left[\begin{array}{llllll}
1 & 0 & 0 & 1 & 1 & 1 \\
0 & 1 & 1 & 1 & 1 & 1 \\
0 & 0 & 1 & 0 & 0 & 0 \\
0 & 0 & 0 & 1 & 1 & 1 \\
0 & 0 & 0 & 0 & 1 & 1 \\
0 & 0 & 0 & 0 & 0 & 1
\end{array}\right]
$$

matrices $U_{X}$ and $D_{X}$ are: 


$$
\begin{aligned}
U_{X} & =\left[\begin{array}{llllll}
2 & 3 & 6 & 3 & 4 & 5 \\
3 & 1 & 5 & 3 & 4 & 5 \\
6 & 5 & 5 & 6 & 6 & 6 \\
3 & 3 & 6 & 3 & 4 & 5 \\
4 & 4 & 6 & 4 & 4 & 5 \\
5 & 5 & 6 & 5 & 5 & 5
\end{array}\right] \\
D_{X} & =\left[\begin{array}{lllllll}
5 & 6 & 6 & 5 & 5 & 5 \\
6 & 5 & 5 & 5 & 5 & 5 \\
6 & 5 & 4 & 5 & 5 & 5 \\
5 & 5 & 5 & 3 & 3 & 3 \\
5 & 5 & 5 & 3 & 2 & 2 \\
5 & 5 & 5 & 3 & 2 & 1
\end{array}\right]
\end{aligned}
$$

Proposition 0.24. Let $X$ be a finite topological space and $M=\left\{x_{i_{1}}, \ldots, x_{i_{m}}\right\}$ a subset of $X$, then

$$
\begin{aligned}
& f_{\mathcal{U}}(M)=n-\sum_{r=1}^{n}\left(\prod_{k=1}^{m} t_{i_{k} r}\right) \\
& f_{\mathcal{D}}(M)=n-\sum_{r=1}^{n}\left(\prod_{k=1}^{m} t_{r i_{k}}\right)
\end{aligned}
$$

Proof.

$$
\begin{aligned}
x_{r} \in M^{\nabla} & \Longleftrightarrow x_{i_{k}} \leqslant x_{r} \text { for all } k=1, \ldots, m \\
& \Longleftrightarrow t_{i_{k} r}=1 \text { for all } k=1, \ldots, m \\
& \Longleftrightarrow \prod_{k=1}^{m} t_{i_{k} r}=1 \\
x_{r} \in M_{\triangle} & \Longleftrightarrow x_{r} \leqslant x_{i_{k}} \text { for all } k=1, \ldots, m \\
& \Longleftrightarrow t_{r i_{k}}=1 \text { for all } k=1, \ldots, m \\
& \Longleftrightarrow \prod_{k=1}^{m} t_{r i_{k}}=1
\end{aligned}
$$

Therefore

$$
\left|M^{\nabla}\right|=\sum_{r=1}^{n}\left(\prod_{k=1}^{m} t_{i_{k} r}\right) \text { and }\left|M_{\triangle}\right|=\sum_{r=1}^{n}\left(\prod_{k=1}^{m} t_{r i_{k}}\right) .
$$

Proposition 0.22 allows to obtain the matrices $U_{X}$ and $D_{X}$ from the topogenous matrix $T_{X}$. Consider now the reverse process of obtaining the topogenous matrix from $U_{X}$ and from $D_{X}$. In our matrix language, (5) and (6) are equivalent to having

$$
t_{i j}=1 \Longleftrightarrow u_{j j}=u_{i j} \Longleftrightarrow d_{i i}=d_{i j}
$$

Therefore, we can reconstruct the topogenous matrix by using the following algorithms:

\section{Algorithm: Topogenous matrix from $U_{X}$}

Input: Matrix $U_{X}=\left[u_{i j}\right]_{n \times n}$ associated to the function $f_{\mathcal{U}}$ of $X$.

Output: Topogenous matrix $T_{X}=\left[t_{i j}\right]_{n \times n}$ associated to the space $X$.

1. for $i=1 \cdots n$

2. for $j=1 \cdots n$

3. $\quad$ if $i>j$ do $t_{i j}=0$, else

4. $t_{i j}=\delta\left(u_{i j}-u_{j j}, 0\right)$

5. end for

\section{6. end for}

\section{Algorithm: Topogenous matrix from $D_{X}$}

Input: Matrix $D_{X}=\left[d_{i j}\right]_{n \times n}$ associated to the function $f_{\mathcal{D}}$ of $X$.

Output: Topogenous matrix $T_{X}=\left[t_{i j}\right]_{n \times n}$ associated to the space $X$.

1. for $i=1 \cdots n$

2. for $j=1 \cdots n$

3. $\quad$ if $i>j$ do $t_{i j}=0$, else

4. $\quad t_{i j}=\delta\left(d_{i j}-d_{i i}, 0\right)$

5. end for

6. end for

Remark 0.25. From proposition 0.24 and above algorithms, we see that if $M=\left\{x_{i_{1}}, \ldots, x_{i_{m}}\right\} \subseteq X$ we have:

$$
\begin{aligned}
& f_{\mathcal{U}}(M)=n-\sum_{r=1}^{n}\left(\prod_{k=1}^{m} \delta\left(f_{\mathcal{U}}\left(x_{r}\right)-f_{\mathcal{U}}\left(x_{i_{k}}, x_{r}\right), 0\right)\right) \\
& f_{\mathcal{D}}(M)=n-\sum_{r=1}^{n}\left(\prod_{k=1}^{m} \delta\left(f_{\mathcal{D}}\left(x_{i_{k}}\right)-f_{\mathcal{D}}\left(x_{i_{k}}, x_{r}\right), 0\right)\right)
\end{aligned}
$$

Therefore, functions $f_{\mathcal{U}}$ and $f_{\mathcal{D}}$ can be calculated for any subset $M$ of the space $X$, from the functions values in the subsets of cardinality one and two through the above explicit formulas. 


\section{Conclusions}

We have seen how the considered matrices make easier the topological study in finite spaces when we use submodular functions, achieving to eliminate the need to draw Hasse diagrams to find its values as was worked in (Abril, 2015) and (Sarria et al., 2014). In future works, it can be studied complexity of the shown algorithms and try to find other topological concepts which could be characterized from this matrix point of view.

Conflict of interest. The authors declare that they have no conflict of interest.

\section{References}

Abril J. A. (2015). Una aproximación a la noción de homotopía entre espacios topológicos finitos desde las funciones submodulares (Trabajo Final de Maestría). Universidad Nacional de Colombia. Facultad de Ciencias. Bogotá, Colombia.
Alexandroff P. (1937). Diskrete Räume. Mat. Sb. (N.S.)2, 501-518.

Cuevas Rozo J. L. (2016). Funciones submodulares y matrices en el estudio de los espacios topológicos finitos (Trabajo Final de Maestría). Universidad Nacional de Colombia. Facultad de Ciencias. Bogotá, Colombia.

Krishnamurthy V. (1966). On the number of topologies on a finite set. Amer. Math. Montly 73, 154-157.

Sarria, H., Roa L. \& Varela R. (2014). Conexiones entre los espacios topológicos finitos, las fd relaciones y las funciones submodulares. Boletín de Matemáticas, 21(1), $31-50$.

Shiraki, M. (1968). On finite topological spaces. Rep. Fac. Sci. Kagoshima Univ., 1, 1-8.

Stong R. E. (1966). Finite topological spaces. Trans. Amer. Math. Soc., 123(2), 325-340. 